\title{
Atomic Force Microscopy: A Multifunctional Tool for Materials Characterization in Shared Resource Centers
}

\author{
Brooke B. Massani
}

Dept. of Chemistry \& Biochemistry and office of Research, Discovery \& Innovation, University of Arizona, Tucson, USA.

Since its invention in 1986 by Binnig et.al. [1] at Stanford University, the Atomic Force Microscope (AFM) has become increasingly "routine" for nanometer-scale characterization of structure and physical/electrical properties of a wide range of both "hard" and "soft" materials. This talk will focus on best practices that we have developed in the W.M. Keck Center for Nanoscale Imaging, where I manage three AFM instruments: Cypher ES, Multimode 8 and Agilent 5500 with 150 users from 45 research groups and 9 departments at the University of Arizona.

Contact mode, the first AFM imaging mode, measures topography by monitoring the deflection of a flexible cantilever with a sharp tip as it raster scans across a sample. A laser focused onto the backside of the cantilever and reflected onto a four-quadrant detector is used to measure the tip-surface interactions. Contact mode can be damaging to soft or weakly bound materials; therefore, soft AFM imaging modes, including non-contact mode in 1987 [2] and Tapping Mode in 1993 [3], were introduced to enable imaging at lower tip-surface interaction forces. Instrumental improvements, development of imaging modes, improved understanding of AFM force measurements, and increasing applications have quickly grown the capabilities of AFM instruments and the imaging field. AFM is a multifunctional technique which operates in air, liquid, and vacuum environments leading to its broad use in materials, engineering, and biological research areas. It is an ideal instrument for shared resource laboratories and/or multiuser environment which can maximize utilization of the instrument to produce quality data for the variety of research applications.

In our facility, the primary goal is to train non-expert researchers to run the systems independently while generating quality AFM measurements for their research application. The most common research questions that the instruments in the Keck Center are used to answer include: what is morphology of my material; what is the thickness of my film; what are the dimensions of objects; and what is the surface coverage. To have confidence in these measurements understanding what makes a good AFM image is essential. For example, images collected using a blunt or broken probe can have artifacts were every object is the shape of the broken probe rather than a true image of the surface as shown in Figure 1. Understanding common image artifacts and the effect of probe shape/contamination on AFM images will be reviewed.

In addition to the common AFM research questions, the Keck Center facilitates pushing the limits of the instruments beyond the manufacturer supplied techniques. Conductive-probe (CAFM) simultaneously measures surface current and topography of a conductive or semi-conductive material when a bias is applied illustrating the conductive heterogeneity of the material (Figure 2). In the current image (Figure $2 \mathrm{~b}$ ) for these types of oxides one can see the spatial heterogeneity in conductivity, thought to arise because of the local compositional variations and distribution of blocking layers that are unavoidably on the oxide surface. Such heterogeneity can have real significance in the performance of devices, such as solar cells and light emitting diodes, which rely on this semi-transparent contact to collect or inject electrical charges into an adjacent active layer. One method going beyond the CAFM technique, used extensively in our 
facility, is mapping of the electrical properties using Scanning Current Spectroscopy (SCS) [4] where a spatially resolved array of current-voltage $(J-V)$ curves are collected, analogous to force-volume maps. The $J-V$ curves are fit to a modified Mott-Gurney expression and used to generate ohmicity maps to determine the quality of electrical contact of electrode, organic and inorganic device materials. Examples of SCS measurements on photovoltaic materials will be presented.

\section{References:}

[1] G. Binnig, C.F. Quate and Ch. Gerber, Physical Review Letters 56 (1986) p930.

[2] Y Martin, C.C. Williams and H.K. Wickramasinghe, Journal of Applied Physics 61 (1987) p4723.

[3] Q. Zhong, D. Inniss, K. Kjoller and V.B. Elings, Surface Science Letters 290 (1993) pL688.

[4] MacDonald, G.A., Veneman, R.A., Placencia, D. and N.R. Armstrong, ACS NANO 6 (2012) p9623.

[5] Samples courtesy of Qingru Wang and Xiushan Zhu.

[6] Samples and images courtesy of James Standfill.

[7] This material is based upon work supported by the National Science Foundation under Grant Number 1337371.
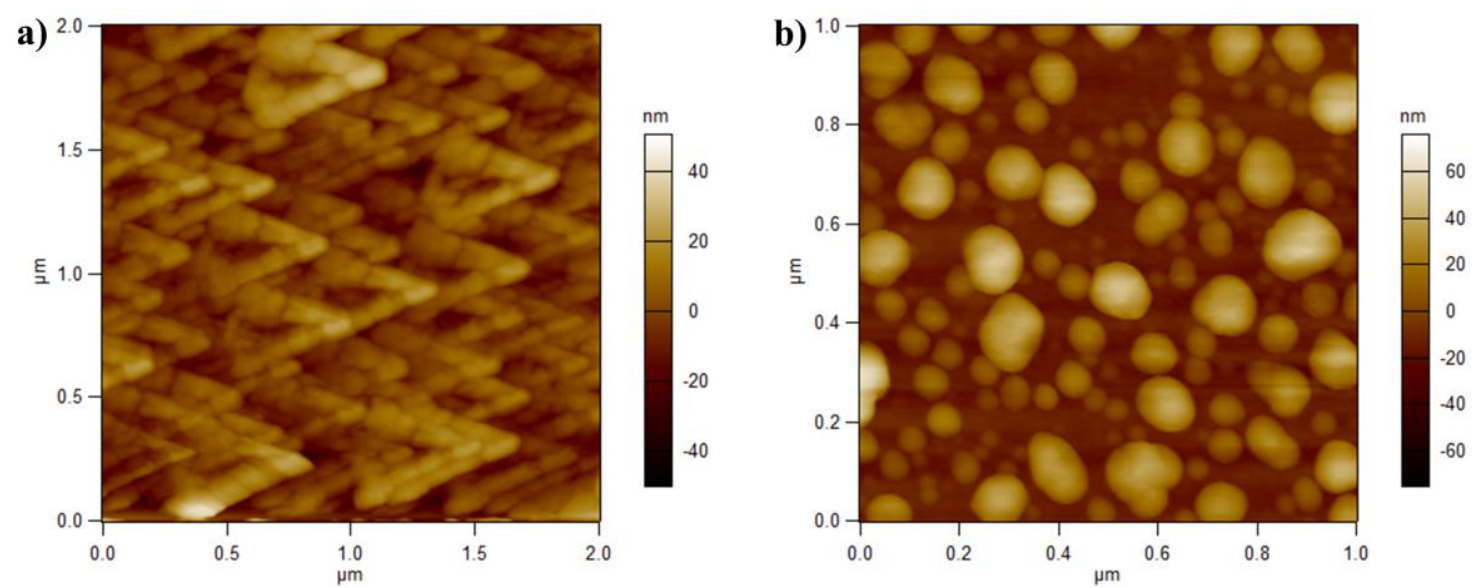

Figure 1. Height images of the same silver-island film with a) a blunt probe and b) a new sharp probe [5] which illustrate AFM image artifacts due to probe wear or damage.
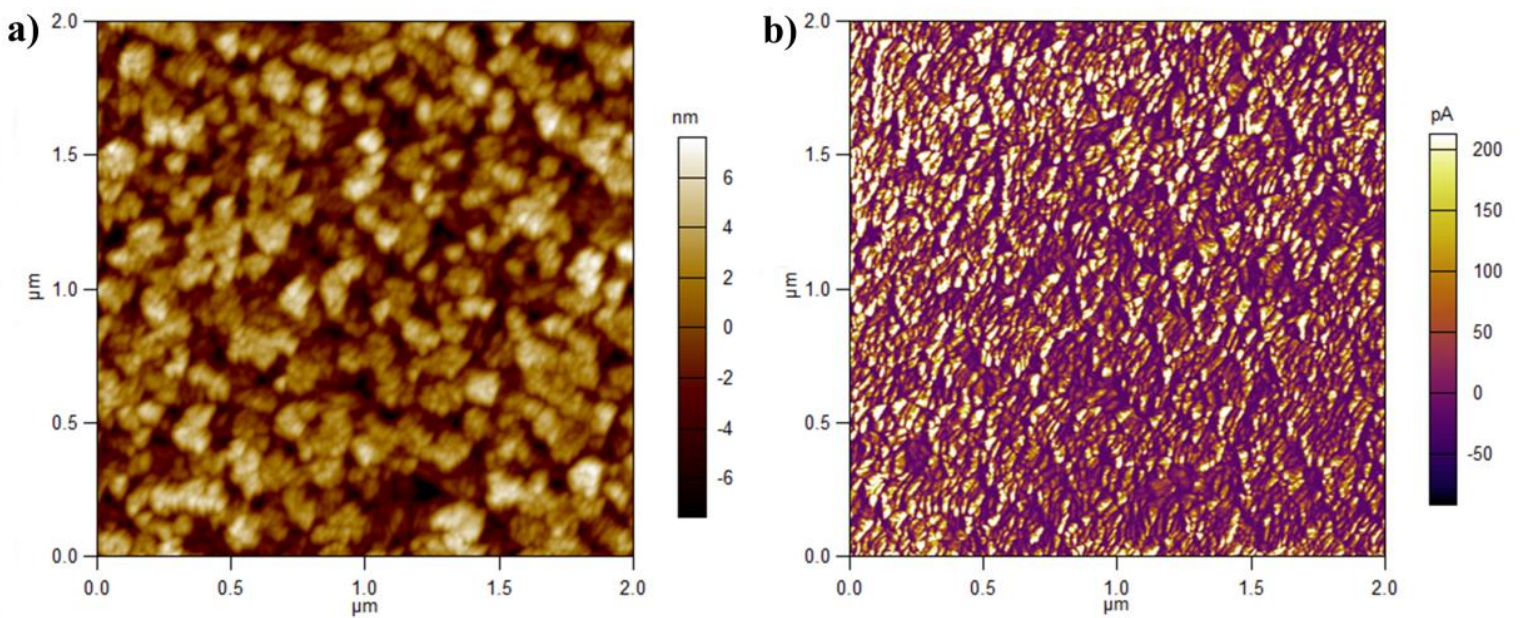

Figure 2. a) Topography and b) current map of indium tin oxide which is a transparent electrode material used in photovoltaic devices [6]. 